\section{GERM INFECTION IN TUBERCULOSIS :}

A SKETCH OF THE PRESENT STATE OF THE QUESTION.

By GEORGE OGILVIE, B.So., M.B.Edin., M.R.C.P.Lond., Physician to the French Hospital, and to the Hospital for Epilepsy and Paralysis, Regent's Park, London.

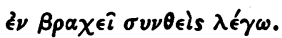

THE evidence in favour of congenital tuberculosis has during the last few years been collected and critically examined independently by a number of authors (Hauser, Mosny, Kuss, Scheimpflug, Dürek, etc ). The common result of their laborious researches is the collection of a very small number of authenticated cases of intrauterine tuberculosis, about 20 in human beings. The number given by Kuss (40) is certainly too high, that admitted by Hauser (18) perhaps a little too low. Of course, this result does not warrant conclusions with regard to the relative frequency of such an occurrence, because almost exclusively positive observations have been published. All that one is entitled to infer from these statistics is the fact that congenital tuberculosis in man is exceedingly rare, a result in perfect accordance with the most extensive post mortem experience of Virchow and others. On the other hand, the appearance of tuberculous disease in subsequent generations is a fact of daily clinical observation. For this reason alone it is not only most probable, but almost certain, that the frequent occurrence of tuberculosis in parent and offspring cannot be accounted for by hereditary transmission of the disease itself. Whether it is entirely due to the increased opportunities for infection (Cornet), or to the hereditary transmission of a diminished resisting power, of a certain predisposition to tuberculous infection, is a controvertible point alien to the subject of this sketch.

The cases hitherto observed present some common features which allow of certain inferences regarding the origin of the fetal infection. In the first place the parental disease was in all cases present in mothers only, or where the father's disease is mentioned the mother was suffering from the disease as well. In cases of the latter kind it is of course impossible to decide with certainty with which of the parents the source of infection has to be sought, while a maternal origin remains by far the most probable. Secondly, in all cases in which the mother's disease is described with sufficient accuracy they were without exception suffering from the most severe forms of tuberculosis, to which they almost all succumbed during pregnancy or shortly after delivery. Thirdly, the affected fetus was in all cases over three months old-that is, at an age when placental circulation has taken the place of the omphalo-mesenteric. Obviously these characteristic traits common to all the cases under discussion point to placental infection. With our present modes of investigation it is impossible to strictly decide whether infection of the fetus by its mother is germinal or placental, unless the latter's infection does not take place until after conception. Yet the evidenee before us favours the presumption that the placental circulation is the way by which infection is carried from mother to fetus in all these cases.

That observations of this kind are inadequate to account for our clinical experience with regard to tuberculous disease in subsequent generations is manifest. In the first place, the father's disease seems, according to clinical evidence, to play as important, or nearly as important, a part in influencing the offspring as that of the mother. Secondly, the disastrous effect upon the progeny is not exclusively, nay, not even proponderatingly, found in the acute and virulent forms of tuberculosis. On the contrary, the lingering and insidious cases of parental disease seem to be those which by preference expose the offspring to the prejudicial effect of tuberculous descent. Thirdly, if the infection of the fetus were germinal one would expect to find evidence of it before the third month of intrauterine life. For these reasons it is out of the question to use this scanty evidence of congenital tuberculosis, evidently due to placental infection, in explanation of the vastly different and much more complex phenomena presented by clinical observation. It may be said without exaggeration that Baumgarten, who with undaunted perseverance maintains this latter view, at present enjoys the doubtful distinction

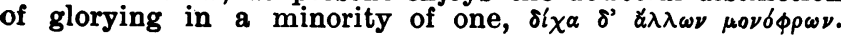

In o.je of his last assurances on this subject, he again gives expression to his unshaken "hope that his views of the parasitic inheritance of tuberculosis will, although slowly, gain more and more ground." " Nothing in the literature of our daps seems to favour this sanguine expectation; in fact, all appearances irdicate a total change in the opposite direction.

It was only natural to appeal to experimental research for the solution of a problem which seemed to baffle the efforts of the clinical observer. It has already been said that for obvious reasons the mode of infection must remain doubtful in almost all cases in which the source of the fetal disease is to be looked for in the mother. While it is certain that postconceptional infection of the mother can find its way to the fetus only by the medium of the vascular circulation, preconceptional or conceptional infection may be communicated to the offspring in this way, as well as by the germ itself, without our being able to decide positively which of the two possible modes has taken place in a given case. Therefore only these experiments could with certainty be interpreted as germinal infection in which positive results were obtained by coupling healthy females with males suffering from tuberculosis. It must be said at once that all the experiments madein this direction which are worthy of serious considerations have, however frequently repeated, answered in the negative. But even if they occasionally yielded a positive result, this would not have gone far to account for the facts of our clinical experience in man, because all the animals were the subjects of the most acute and disseminated forms of general virulent tuberculosis. It was, therefore, an appropriate idea to repeat the experiments in rabbits and guinea-pigs, which were made experimentally to suffer from mild, initial, and localized forms of tuberculous disease. Here, again the results were all negative, with one solitary exception, in which tuberculosis of the liver, the mesenteric and retroperitoneal glands were found in the offspring, whilst the most careful necropsy of the mother revealed no trace of tuberculous disease. The author (Hauser) cautiously and judiciously attributes this apparently positive result to accidental infection by feeding, in favour of which mode of infection he adduces the participation of the mesenteric glands, and also the circumstance that an easy possibility of such infection was afforded by the fact that the attendant who had care of the animal was at the time suffering from severe tuberculous disease. Baumgarten cites this result as " an undoubtedly positive one," in opposition to the author's interpretation and to the facts of the case.

It now remains to mention Baumgarten's and Maffucci's well-known experiments, the demonstrative power of which the former still defends in his last discussion of the question (1900). By injecting tubercle bacilli into hen's eggs, chickens were produced affected with tuberculous disease. By this fact the authors believed to have proved that a germ cell containing bacilli may develop into an individual affected with the bacillary disease. This conclusion disregards two points of essential importance. In the first place it is not pruved that a germ cell containing a living bacillus will ever reach that stage of development in which it is possible to inject a culture of bacilli into it. The argument overlooks, and consequently confounds, the different stages of development of the orum from the initial germ to the "chicken i' the shell." The fallacy is akin to that which Sir John Falstaff was guilty of when he objected to "pullet-sperm in his brewage." Secondly -and this is the most important point-experiments made on bird's eggs allow of no extension or application to the human germ cell. It is true that a bird's egg represents only one cell, but in an advanced stage of development, and of a totally different structure from that of the mammal. Strauss in his excellent work on the tubercle bacillus had already observed that in the experiments the inoculation was made into the zolk, or the white of the egg, but not into the germ proper. It is quite possible that bacilli may develop and multiply in this manner, and later on infect the germ through the vascular area, in the same way as the embryo of the animal is infected through the placenta. To bring these experiments to the same level with the infection of the mammalian ovum it would have been necessary to inject the tubercle bacilli into the germinal vessel, and not under the egg's shell. In structure and development the holoblastic 
ovum of the mammal so materially differs trom the meroblastic ovam of the bird that biological properties of the latter cannot without further examination be attributed to the former (Hauser). Baumgarten in his last reply turns the whole argument topsy-turvy by asking "how one is to know that the bacilli were not injected into the germinal vessel ?" To give his experiments the conclusiveness which he claims for them it would have been his task to prove that the bacilli were actually introduced into the germ proper. This he is unable to do. Until such proof is given, the indirect infection of the germ will be coneidered by far the more probable process. "Anyhow," he continues, "the bacilli were present in the chicken which develops neither out of the yolk, nor out of the white, but out of the germ." This again of course proves nothing, for infection through the vascular area is analogous to placental infection, and the existence of the disease in the newborn no more proves germinal infection than congenital syphilis of the infant of a mother suffering from post-conceptional syphilis proves germinal infection.

For exactly the same reason. namely, the fundamental difference between the holoblastic and the meroblastic ovum, it is impossible to extend to human pathology the experience made with pebrine. This disease seems to be the only one in which, up to the present, germinal infection has been pathologically proved beyond legitimate doubt. The sexual organs are invaded by the protozoon, and thus the latter is introduced into the germ. The principal source of infection are the faeces of diseased silkworms which develop out of the infected eggs. The practical result of Pasteur's ingenious proposal to examine microscopically the egge, and to destroy those which were found to contain the sporozoon, has afforded additional proof of the existence of germinal transmission in this particular affection. But here again it has to be borne in mind that the insect's ovum is of meroblastic nature, that pathological germs situated in the central yolk need not interfere with the peripheral development of the embryo, and for this reason alone the argument taken from pebrine can never be used in support of germinal infection in man.

As an irrefutable instance of the latter syphilis is frequently given. Baumgarten, in particular, again and again insists upon the "history of syphilis" as bearing " unexceptional proof that the holoblastic germ may, in spite of invasion by the microbe of syphilis, develop into a full-term well formed child." Unfortunately this much-hunted-for microbe is still a desideratum, its development or life-history consequently a blank. Paternal syphilitic infection may be, and evidently is to some minds a clinical postulate; but between this and an " unexceptionable" truth demonstrated and established by pathological evidence there is a wide gulf. Moreover, what at one time to one mind may be a clinical postnlate, may not prove so to other minds, or even to the same mind at other times. Of this psychological fact I know no more striking illustration than that of P. Diday, the eminent Lyons syphilographer, at one time the most enthusiastic, energetic, and eloquent champion of "paternal infection," choc en retour" and "imperceptible syphilization" of the mother. These doctrines he proclaimed in his Paris Lectures (1863) as irrevocable truths, as the inevitable logical consequences of undeniable facts. Twenty-five years later, as may be seen from his last lecture delivered in Paris, these same doctrines had so completely lost all hold upon his sense of logical cogency, that he revoked them one and all, and professed with equal enthusiasm, energy, and eloquence the opposite creed : Nihil est in foetu quod non prius fuerit in matre. The pathology of syphilitic heredity is so entirely conjectural, and has, apart from an abundance of theories, gained so little by the modern progress in the knowledge of infectious diseases that it is least able to support " by analogy" a questionable cause.

If thus proof of the reality of germinal infection in man has not been forthcoming, the possibility of such an occurrence cannot be denied on $a$ priori theoretical considerations, if only the bacillus of the disease in question can be traced in the seminal fluid. With regard to tuberculosis this seems to have been accomplished with certainty. Cases complicated with tuberculous disease of the testis and epididymis have, of course, to be excluded. There remains, however, a number of positive observations, the reliability of which is scarcely to be doubted, although it has to be taken into account that in investigations of this kind there is no small danger of accidental infection, and that for this reason negative results are perhaps of greater weight than positive statements. Walther is the only observer who in 12 cases of chronic pulmonary phthisis examined the testes, epididymes, the prostatic gland and the contents of the seminal vesicles with purely negative results. The negative results obtained by Rohlff are explainable by the insufficient quantity of sperma introduced into the anterior chamber of the eye of the rabbit, also by the circumstance that the animals were not kept alive for a sufficiently long period. On the other handthe conclusiveness of the positive results granted-it has to be remembered that here, again, the individuals in whom sperm bacilli were found were always suffering from severe fatal tuberculosis, mostly tuberculosis of the lungs partly combined with tuberculosis in numerous other organs, even kidneys and prostatic glands. In the ordinary course of tuberculous "heredity" as it presents itself to clinical obsevation, the conditions are totally different.

Gaertner, ${ }^{2}$ who himself has obtained positive results with regard to the presence of the bacilli in the semen of tuberculous guinea-pigs, tries to prove the infinitesimal chance of germinal paternal infection by the following remarkable computation. Assuming that the tubercle bacillus can reach the ovum only through conveyance by a spermatozoon, he calculates the chances of a simultaneous occurrence of impregnation and infection. In a human ejaculation the number of spermatozoa amounts, according to Lode, ${ }^{3}$ to about 225,000,000, while Gaertner supposes-and this is in his opinion a high estimate-that it may contain from ro to roo tubercle bacilli. In guinea-pigs which are in a condition of health most favourable to this purpose of 6.5 ejaculations, only one is tuberculous. This transferred to man will give Io to 100 tubercle bacilli to $6.5 \times 225=1462.5$ millions of spermatozoa. Suppose even that each copulation leads to impregnation, the chance of infection would be, at the best, I00: $1462.5=1 ; 14.625$ millions. Arguments appearing in the armour of mathematical deduction seem to be proof against criticism. Yet doubts must be raised with regard to the hypothetical premisses on which this algebraic structure is reared. It does not seem self-evident that the bacillus can reach the female germ only through conveyance by a spermatozoon. It is not impossible that the act of copulation, particularly when it leads to impregnation, induces some active absorbing motion in the female genital tract which conveys the seminal fluid en masse, and without self-exertion on the part of the spermatozoa, to the seat of the ovum (Scheimpflug). It may also be that the alterations of pressure caused by the mechanism of ejaculation lead to an increased passage of bacilli into the sperma of a patient in whose blood and sexual organs these organisms are moving. There are so many more possibilities hardly to be foreseen by theory, much less to be put into calculative account, that speculations of this kind, however mathematically dressed, will scarcely carry conviction. What we require is experimental evidence, and this it seems has at last been afforded by some recent researches to which I wish to draw special attention.

Dr. F. Friedman 4 experimented on doe rabbits which were put together with bucks immediately, or, at the latest, a few hours after delivery. This, according to his experience, is the only period at which in rodents, and in rabbits particularly, copulation leads with certainty to conception and gravidity. Directly after coition one or two drops of a thin emulsion of tubercle bacilli in a sterile and slightly alkaline solution of hydrochlorate of soda were injected into the vagina. The solution contained a small lump of fresh virulent culture of less than $1 \mathrm{~mm}$. diameter to 2 to $3 \mathrm{ccm}$. of fluid motter. The syringe used bore a mouthpiece about $3 \frac{1}{2} \mathrm{~cm}$. long, which length corresponds to that of the erect penis of the rabbit. On examining about an hour later the mucus gathering at the exterior mouth of the womb, large quantities of spermatozoa were found and only isolated tubercle bacilli. ${ }^{5}$ The female animals were killed within a week after the performance of the experiment. In all the embryos tubercle bacilli were found. Their number varied considerably with different mothers, and also with different embryos of the same mother. It is remarkable that the number of places in which tubercle 
bacilli were found vary in different individuals belonging to the same pregnancy from 2 in one to 26 in another. Not a single tubercle bacillus was found by microscopical examination of the mucous membrane of the uterus or the vagina. In the open lumen of the uterus twice only an isolated bacillus was detected in close neighbourhood of the embryo. Of course no pathological changes of the maternal organs due to the tubercle bacillus could be expected in so short a period, but there was every reason to expect numerous tubercle bacilli in the open lumen of the uterus, or, at least, adjacent to the mucous membranes of uterus and vagina. This, however, is not the case, and it seems, strange as it sounds, that all bacilli which do not make their way into the ova are eliminated from the genital tract. The mode by which the bacilli reach the female cell has not yet been dis. covered. To decide the question whether tubercle bacilli can be carried into the ovum by the spermatozoon, experiments are in operation at present with Ascaris megalocephala. But for the decision of the general question it is irrelevant whether bacilli introduced together with the semen reach the ovum at the very moment of impregnation, or shortly before or after it.

Further experiments will have to demonstrate how the bacilli develop in the embryo, at what time the first anatomical and histological signs of tuberculosis become apparent, and whother the mother remains healthy, or becomes infected in her turn (choc en retour). It will also have to be shown in embryos at the earliest stages before the formation of a placenta, that males rendered tuberculous by intraperitoneal, intratracheal, intraocular, etc., injection produce infected offspring, while the mother remains immune. It may be added that experiments in which tubercle bacilli were introduced into the bladder by way of catheter have given negative results. The same result followed after the injection into the vagina of the semen of fresh killed males, which had been previously mixed with a culture of tubercle bacilli. The author is aware that his mode of experimental procedure does not correspond with the natural course of things, in particular that, although one or two drops of a very thin emulsion were injected, the sperma contained a great many more bacilli than are ever to be found in the semen of a phthisical patient. Yet these experiments seem to prove that tubercle bacilli introduced into the vagina, together with the semen, may pass into the embryo without the intermediary of a mother. Of course it is a question altogether different how frequently, if at all, this takes place in human tuberculosis. On this point we know nothing.

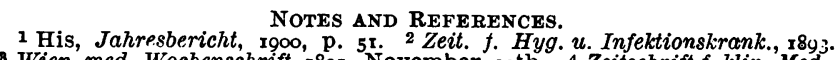
Wien. med. Wochenschrift, r89x, November 20 th. 4 Zeitschrift f. klin. Med. xiii, rgor. These experiments have been made under the supervision of Professor Gerbard, the well-known teacher of clinical medicine. Pro等 remarked that experiments made on ouinea-pigs or berlin. remarked that experines rats, and white mice hover the semen, a viscous thick matter, which introduced into the vagin congeals into a hard plug, completely flllng up the vagina up to the uterus. The general opinion that this plug prevents the outflow of th removal of the plug for the introduction of the be correct, because afte take place.

\section{THE USE OF PARAFFIN}

FOR RESTORING THE BRIDGE OF THE NOSE. By STEPHEN PAGET, F.R.C.S.,

Surgeon to the West London Hospital; Surgeon to the Ear and Throat Department of the Middlesex Hospital.

IT seems likely that the subcutaneous injection of paraffin, after the method discovered by Gersuny, is a very great improvement on all other treatments in cases where the bridge of the nose is sunken by injury or disease. The simplicity of the method, and the excellent result that is at once produced, are in strong contrast with the difficult, tedioss, and uncertain course of plastic operations, and of all attempts to use metal, celluloid, and the like substances. There is indeed something almost absurd in the mere injection of the paraffin, and the immediate restoration of a fairly good profile to a face that has been unhandsome for years; and the novelty of the method may justify thiz note on two cases where it was used.
The first case was that of a student, whose nose had been badly broken by a fall when he was 7 years old. There was an old fracture of the septum, but no marked nasal obstruction and no lateral deviation of the nose. But the whole middle portion of the nose was sunken, and the lower portion was broad and ill shaped, with the nostrils circular and looking rather forward. Under an anaesthetic the septum was refractured and straightened, a Walsham's nasal splint was applied to it, and a small quantity-about 2 c.cm.-of paraffin was injected under the skin in the middle line.

The second case was that of a man about 30, suffering from laryngeal phthisis, whose nose was sunken from disease, probably syphilitic, many years ago. In this case the interior of the nose was left untouched, and, under an anaesthetic, about $4 \mathrm{c} . \mathrm{cm}$. of paraffin was injected under the skin.

The result in both cases was very satisfactory ; the profile is restored, and the patient is pleased at his improved looks. Of course, in cases of this kind, there are faults that cannot be corrected either by Gersuny's method or by any other. A nose that is too short cannot be lengthened, and a nose that is too broad across the nostrils cannot be diminished in breadth. But the advantage of the paraffin treatment is that it accomplishes, in a few minutes, without a scar, more than plastic surgery can ensure by a severe operation. With a needle and a syringe and a bottle of paraffin, the surgeon can, in five minutes, remedy that sunken deformity of the nose which is so distressing to a man, not only on account of its unsightliness, but because it is taken for granted as positive evidence of syphilitic disease.

In the first case, a paraffin (Eckstein's) was used, that had a melting-point of $136^{\circ} \mathrm{F}$. In the second, a paraffin with a melting-point of $115^{\circ}$. The following considerations are to be noted :

1. Whatever may be the melting point of the paraffin, it must be kept, during use, $10^{\circ}$ or $15^{\circ}$ higher, or it will solidify in the needle before it can be injected.

2. For the same reason, the needle and syringe must be kept $15^{\circ}$ or $20^{\circ}$ higher than the paraffin. The loss of heat from the syringe may be to some extent checked by casing it in a bit of drainage tube.

3. An ordinary glass antitoxin syringe, with a well-fitting asbestos piston, answers every purpose. The needle must be broad and strong, such as is used for exploring the pleural cavity; but the needle generally used for this purpose is too long, and should be shortened to an inch and a half.

4. An assistant must make firm pressure, very carefully, all round the nose, and must keep up this pressure till the paraffin is set. But it sets almost at once, allowing only a quarter or half of a minute to the surgeon to mould it. The firm pressure may be helped by the use of a strip of lead or pewter under the tips of the fingers.

5. The skin of the nose, at the point where the needle is to enter, should be just nicked with a scalpel, so that the needle may pass easily. It is best to direct the needle downward, away from the eyelids, and to introduce it at the middle line of the nose.

6. Eckstein's paraffin, melting at $136^{\circ}$, is difficult to use, and must be very quickly transferred from the bottle into the subcutaneous tissue. Probably it is best suited for a case where only a very small quantity of paraffin is required. With this paraffin the syringe must be kept so hot that it can hardly. be handled except with gloves.

7. One case has been recorded of sloughing of the skin, presumably from heat. This disaster coula hardly happen with paraffin melting at $115^{\circ}$. More than one case has been recorded where signs attributable to pulmonary embolism followed the injection. It is therefore necessary to avoid piercing a vein, and to keep firm and close pressure all round the nose during and after the injection.

8. After the treatment a fold of lint should be kept over the upper part of the face, and kept moist with cold or iced lotion. In the two cases quoted there was little or no pain after the operation, but some swelling round the nose for three or four days.

It is, of course, impossible by any method to make a narrow or refined bridge to the nose in these eases. The front view of it is still heavy and clumsy, but the profile view is excellent, and this good result is obtained without any scar and without any severe or difficult operation, in a fow 